\title{
Stationary uphill currents in locally perturbed zero-range processes
}

\author{
Emilio N. M. Cirillo ${ }^{1, *}$ and Matteo Colangeli ${ }^{2, \dagger}$ \\ ${ }^{1}$ Dipartimento di Scienze di Base e Applicate per l'Ingegneria, Sapienza Università di Roma, via A. Scarpa 16, I-00161, Rome, Italy \\ ${ }^{2}$ Dipartimento di Ingegneria e Scienze dell'Informazione e Matematica, Università degli Studi dell'Aquila, via Vetoio, 67100 L'Aquila, Italy
}

(Received 11 September 2017; published 27 November 2017)

\begin{abstract}
Uphill currents are observed when mass diffuses in the direction of the density gradient. We study this phenomenon in stationary conditions in the framework of locally perturbed one-dimensional zero range processes (ZRPs). We show that the onset of currents flowing from the reservoir with smaller density to the one with larger density can be caused by a local asymmetry in the hopping rates on a single site at the center of the lattice. For fixed injection rates at the boundaries, we prove that a suitable tuning of the asymmetry in the bulk may induce uphill diffusion at arbitrarily large, finite volumes. We also deduce heuristically the hydrodynamic behavior of the model and connect the local asymmetry characterizing the ZRP dynamics to a matching condition relevant for the macroscopic problem.
\end{abstract}

DOI: 10.1103/PhysRevE.96.052137

\section{INTRODUCTION}

Fick's law of diffusion stands as one of the basic tenets of the theory of transport phenomena and irreversible thermodynamics, and predicts that mass diffuses against the density gradient $[1,2]$. Nonetheless, there is some increasing experimental and theoretical evidence, in the literature, of diffusive currents flowing from a reservoir with lower density towards one with larger density, that are hence said to go uphill [3-6]. Such "anomalous" currents have been observed and studied in different contexts. Consider, for instance, a system made of particles of a certain species $A$, whose diffusive motion obeys the standard Fick's law, namely, the current of particles $A$ includes a term proportional to minus the density gradient of $A$ itself. Suppose that a second species $B$ is then added, whose interaction with $A$ affects the diffusive motion of the particles of the first species. Thus, a second contribution to the current of particles $A$ arises, related to the density gradient of $B$, that may counterbalance the first contribution. As a result, at variance with the standard Fick's law prescription [7,8], the species $A$ undergoes a process of uphill diffusion induced by the external potential generated by the species $B$ : this is, essentially, the phenomenon highlighted in the seminal paper by Darken [9], reporting an experiment of transient diffusion of carbon atoms subjected to a repulsive interaction with silicon particles in a welded specimen, where the silicon content is concentrated on the left of the weld (and negligible on the right).

A second stationary mechanism which is known to produce uphill currents is related to the presence of a phase transition in nonequilibrium conditions $[10,11]$. This phenomenon has been observed in computer simulations in a model constituted by a single species undergoing a liquid-vapor phase transition. This system, with one boundary fixed at the density of the metastable vapor phase and the other at the density of the metastable liquid phase, exhibits a stationary state in which the current flows from the vapor boundary to the liquid one. In particular, in Ref. [12] the authors prove the existence of the uphill diffusion phenomenon for a stochastic cellular

\footnotetext{
*emilio.cirillo@uniroma1.it

${ }^{\dagger}$ matteo.colangeli1@univaq.it
}

automaton, in which the particles are subjected to an exclusion rule (preventing the simultaneous presence of two particles with same velocity on a same site) and to a long-range Kac potential [13]. As distinct from the Darken experiment, the mechanism responsible, in this case, for the breaking of the standard diffusive behavior is the creation of a sharp interface located near one of the two boundaries, called a bump therein, separating the vapor and the liquid phases. The density profile results essentially decreasing almost everywhere along the one-dimensional (1D) spatial domain, except at the transition region: in fact, the stationary current proceeds downhill in most of the space, but it goes uphill right along the interface.

Noticeably, the occurrence of stationary uphill currents induced by a phase transition was also recently reported in Ref. [14], for a two-dimensional Ising model in contact with two infinite reservoirs fixing the values of the density at the horizontal boundaries.

In this paper we study a different mechanism to produce uphill currents, based on a local perturbation of a stationary state. The model discussed allows us to recover some of the important features of the physical examples of uphill diffusion mentioned above. In fact, despite being simple enough to permit an analytical solution, it gives rise to a stationary uphill diffusion which is not induced by a phase transition as in Refs. [11,12,14], but is triggered by a local asymmetry in the hopping rates that rule the microscopic dynamics in the bulk. The asymmetry at the center of the lattice stands as a caricature of the external potential exerted by the silicon particles on the carbon atoms, as described in the Darken experiment [9]; cf. also the setup discussed in Sec. III of Ref. [15].

The effect of local perturbations of stationary states is a fascinating problem which received much attention in the recent physics and mathematical literature; see, e.g., the review [16]. A classical question in this field is the so-called blockage problem, posed in Ref. [17] for the totally asymmetric simple exclusion process on a ring. The question is whether slowing down a single bond on the lattice can ultimately affect the value of the stationary current in the infinite volume limit; see also Refs. [18-20] for related results for different models.

Differently from the blockage problem, the question addressed in this paper concerns the effect of a local asymmetry in a globally symmetric model. Consider the stationary state 
of a 1D system with symmetric dynamics and suppose that a nonvanishing current exists due to the coupling of the system with two particle reservoirs at the boundaries. What happens if the dynamics is perturbed and made asymmetric just on a single site of the lattice? Is such a local asymmetry effective enough to reverse the natural current flowing direction?

More precisely, the model we shall consider is a $1 \mathrm{D}$ channel with open boundaries at its extremities [hereafter called a zero range process-open boundary (ZRP-OB)], in contact with two reservoirs. The reservoirs are equipped with assigned particle densities, which also fix the injection rates at the boundaries. The dynamics in the channel is symmetric, therefore in the steady state a particle current exists which moves from the reservoir with larger density to the one with smaller density, as prescribed by the Fick's law. Then, on a single site at the center of the lattice, the dynamics is modified in such a way that particles locally hop with a higher rate towards the reservoir with larger density. More general inhomogeneous random ZRPs have been considered in the recent literature $[21,22]$. We prove that such a bias may give rise to stationary uphill currents in the channel. In particular, we prove that for any fixed difference between the two injection rates it is always possible to tune the local asymmetry in order to observe an uphill current for arbitrarily large finite volumes. The mechanism is the following: for sufficiently large volumes the density at the boundaries of the channel depends only on the injection rates and not on the local bias; moreover, if the bias is large enough the current changes sign so that the particles move uphill. The model we shall use is a $1 \mathrm{D}$ ZRP. More detailed results will be derived by establishing an appropriate form for the intensity function, namely the rate at which a site is updated, and eventually this will be chosen proportional to the number of particles occupying the site. In this case, we shall also develop a heuristic argument to derive the hydrodynamics equations. These will be endowed with two matching conditions-one concerning the density function and another its first space derivative-at the center of the slab, stemming from the local asymmetry in the hopping rates characterizing the microscopic dynamics. We will then solve the problem via a Fourier series expansion, and we shall finally compare, finding a perfect match, the solution of the hydrodynamic problem with the evolution of the original ZRP. We also mention that uphill currents are observed in queuing network models.

Moreover, we will introduce a periodic version of the inhomogeneous ZRP, in which the channel is coupled at its extremities with two slow sites, mimicking two finite particle reservoirs, which can also exchange particle between themselves: the whole system thus constitutes a closed circuit (hereafter called a ZRP-CC). One of the open questions posed in Ref. [12], in the context of stochastic particle systems, was the conjectured existence of stationary states with nonvanishing self-sustained currents running in circuits, this phenomenon also being called "time crystals" in the literature $[23,24]$. We shall not tackle rigorously the existence of those fascinating rotating states here; rather, we aim to give theoretical and numerical evidence that the local asymmetry introduced in the ZRP-CC may lead to a stationary state in which the densities of the finite reservoirs are different and the current flows, in the channel, from the reservoir with lower density to the one with larger density (as was also the case for the ZRP-OB). Steady states for ZRPs with periodic boundary conditions and spatially varying hopping rates were also discussed in Refs. [25,26].

The paper is organized as follows. In Sec. II we introduce the two ZRP models, the ZRP-OB and the ZRP-CC, and we define the stationary current and recall some useful properties. In Sec. III we prove the existence of uphill currents for the ZRP-OB model. Section IV is devoted to the study of uphill currents for the ZRP-CC. In Sec. V we discuss heuristically the hydrodynamic limit of the ZRP-OB and compare the solution of the hydrodynamic equation to the profile evolving according to the stochastic ZRP dynamics. Finally, Sec. VI is devoted to our brief conclusions.

\section{THE MODEL}

We define the two ZRP models to be studied in the following sections; see also Refs. [27-29] for a survey on ZRP models.

\section{A. The ZRP-OB}

We consider a positive integer $R$ and define a ZRP on the finite lattice $\Lambda=\{1, \ldots, 2 R+1\} \subset \mathbb{Z}$. We consider the finite state or configuration space $\Omega_{R}=\mathbb{N}^{\Lambda}$. Given $n=$ $\left(n_{1}, \ldots, n_{2 R+1}\right) \in \Omega_{R}$ the non-negative integer $n_{x}$ is called number of particles at the site $x \in \Lambda$ in the state or configuration $n$. We let $u: \mathbb{N} \rightarrow \mathbb{R}_{+}$, a positive and nondecreasing function such that $u(0)=0$, be the intensity. Given $n \in \Omega_{R}$ such that $n_{x}>0$ for some $x=1, \ldots, 2 R+1$, we let $n^{x, x \pm 1}$ be the configuration obtained by moving a particle from the site $x$ to the site $x \pm 1$; in particular, we understand $n^{1,0}$ and $n^{2 R+1,2 R+2}$ to be the configurations obtained by removing a particle from the site, respectively, 1 , and $2 R+1$. Similarly, we denote by $n^{0,1}$ and $n^{2 R+2,2 R+1}$ the configurations obtained by adding a particle to the site 1 and $2 R+1$, respectively.

Given $p, q, \bar{p}, \bar{q}, \alpha, \beta, \gamma, \delta>0$ we set $q_{1}=\gamma, q_{x}=q$ for $x=2, \ldots, R$ and $x=R+2, \ldots, 2 R+1, q_{R+1}=\bar{q}, p_{x}=p$ for $x=1, \ldots, R$ and $x=R+2, \ldots, 2 R, p_{R+1}=\bar{p}$, and $p_{2 R+1}=\beta$.

We then consider the ZRP-OB model, defined as the continuous time Markov jump process $n(t) \in \Omega_{R}, t \geqslant 0$, with rates

$$
r\left(n, n^{0,1}\right)=\alpha \text { and } r\left(n, n^{2 R+2,2 R+1}\right)=\delta
$$

for particles injection at the boundaries, and with rates

$$
r\left(n, n^{x, x-1}\right)=q_{x} u\left(n_{x}\right) \text { for } x=1, \ldots, 2 R+1
$$

for bulk leftwards displacements, and

$$
r\left(n, n^{x, x+1}\right)=p_{x} u\left(n_{x}\right) \text { for } x=1, \ldots, 2 R+1
$$

for bulk rightwards displacements (see Fig. 1). Note that Eqs. (2) and (3) for $x=1$ and $x=2 R+1$, respectively, account for the particles' removal at the boundaries. The 


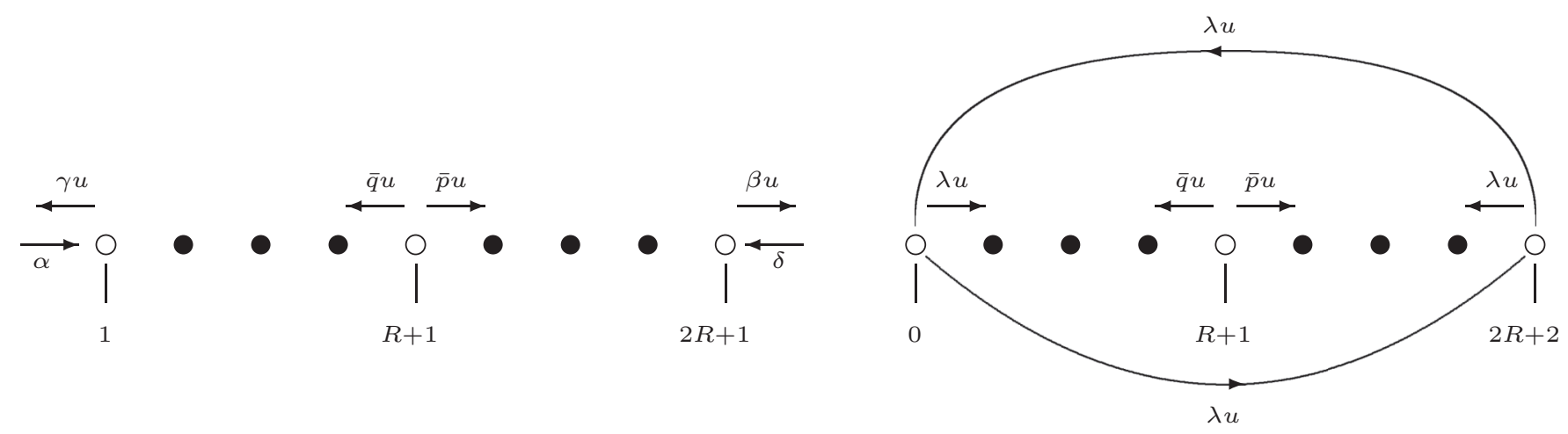

FIG. 1. Schematic representation of the ZRP-OB model (left panel) and the ZRP-CC model (right panel). Rates associated with the black sites are $p u$ towards the left and $q u$ towards the right.

generator of the dynamics can be written as

$$
\begin{aligned}
\left(L_{R} f\right)(n)= & \alpha\left[f\left(n^{0,1}\right)-f(n)\right] \\
& +\sum_{x=1}^{2 R+1}\left\{q_{x} u\left(n_{x}\right)\left[f\left(n^{x, x-1}\right)-f(n)\right]\right. \\
& \left.+p_{x} u\left(n_{x}\right)\left[f\left(n^{x, x+1}\right)-f(n)\right]\right\} \\
& +\delta\left[f\left(n^{2 R+2,2 R+1}\right)-f(n)\right]
\end{aligned}
$$

for any real function $f$ on $\Omega_{R}$.

This means that particles hop almost everywhere on the lattice to the neighboring sites with rates $q u\left(n_{x}\right)$ and $p u\left(n_{x}\right)$. At the center of the lattice, instead, different rates are assumed, namely, $\bar{q} u\left(n_{x}\right)$ and $\bar{p} u\left(n_{x}\right)$. The system is "open" in the sense that a particle hopping from the sites 1 or $2 R+1$ can leave the channel via, respectively, a left or a right move, with rates $\gamma u\left(n_{1}\right)$ and $\beta u\left(n_{2 R+1}\right)$. Finally, particles are injected in the channel at the left and right boundaries with rates, respectively, $\alpha$ and $\delta$.

No further characterization of the (infinite) reservoirs is required for the $\mathrm{ZRP}-\mathrm{OB}$, as the action of the reservoirs is suitably described in terms of the injection rates $\alpha$ and $\delta$. Nevertheless, it may be useful to think of each injection rate as being proportional to the (fixed, for the ZRP-OB model) particle density of the corresponding reservoir, as proposed in Ref. [14] for a continuous-time dynamics; see also Refs. [11,12] in the case of a cellular automaton. Hence, a larger injection rate corresponds to a larger density of the reservoir.

\section{B. The ZRP-CC}

The definition of the model is similar to the ZRP-OB. We consider the positive integers $R, N$ and define a ZRP on the finite torus $\Lambda=\{0,1, \ldots, 2 R+2\} \subset \mathbb{Z}$. We consider the finite configuration space $\Omega_{R, N}=\left\{n \in\{0, \ldots, N\}^{\Lambda}, \sum_{x \in \Lambda} n_{x}=\right.$ $N\}$. Given $n=\left(n_{0}, \ldots, n_{2 R+2}\right) \in \Omega_{R, N}$ the non-negative integer $n_{x}$ is called number of particles at the site $x \in \Lambda$ in the configuration $n$. We let $u: \mathbb{N} \rightarrow \mathbb{R}_{+}$, a positive and nondecreasing function such that $u(0)=0$, be the intensity. Given $n \in \Omega_{R}$ such that $n_{x}>0$ for some $x=0, \ldots, 2 R+2$, we let $n^{x, x \pm 1}$ be the configuration obtained by moving a particle from the site $x$ to the site $x \pm 1$, where we denote by $n^{0,-1}$ the configuration obtained by moving a particle from the site 0 to the site $2 R+2$, and by $n^{2 R+2,2 R+3}$ the configuration obtained by moving a particle from the site $2 R+2$ to the site 0 .

Given $p, q, \bar{p}, \bar{q}, \lambda>0$ we set $q_{x}=q$ for $x=1, \ldots, R$ and $x=R+2, \ldots, 2 R+1, q_{R+1}=\bar{q}, p_{x}=p$ for $x=1, \ldots, R$ and $x=R+2, \ldots, 2 R+1$, and $p_{R+1}=\bar{p}$. We consider the periodic ZRP defined as the continuous time Markov jump process $n(t) \in \Omega_{R}, t \geqslant 0$, with rates

$$
r\left(n, n^{0, \pm 1}\right)=\lambda u\left(n_{0}\right)
$$

and

$$
r\left(n, n^{2 R+2,2 R+2 \pm 1}\right)=\lambda u\left(n_{2 R+2}\right)
$$

for the boundary conditions, and with rates

$$
r\left(n, n^{x, x-1}\right)=q_{x} u\left(n_{x}\right) \text { for } x=1, \ldots, 2 R+1
$$

for bulk leftwards displacements, and

$$
r\left(n, n^{x, x+1}\right)=p_{x} u\left(n_{x}\right) \text { for } x=1, \ldots, 2 R+1
$$

for bulk rightwards displacements (see Fig. 1). The generator of the dynamics can be written as

$$
\begin{aligned}
\left(L_{R, N} f\right)(n)= & \lambda u\left(n_{0}\right)\left[f\left(n^{0,-1}\right)-f(n)\right] \\
& +\lambda u\left(n_{0}\right)\left[f\left(n^{0,1}\right)-f(n)\right] \\
& +\sum_{x=1}^{2 R+1}\left\{q_{x} u\left(n_{x}\right)\left[f\left(n^{x, x-1}\right)-f(n)\right]\right. \\
& \left.+p_{x} u\left(n_{x}\right)\left[f\left(n^{x, x+1}\right)-f(n)\right]\right\} \\
& +\lambda u\left(n_{2 R+2}\right)\left[f\left(n^{2 R+2,2 R+1}\right)-f(n)\right] \\
& +\lambda u\left(n_{2 R+2}\right)\left[f\left(n^{2 R+2,2 R+3}\right)-f(n)\right]
\end{aligned}
$$

for any real function $f$ on $\Omega_{R}$.

The ZRP-CC model differs from the ZRP-OB for the boundary conditions: particles can neither exit nor enter the system. Furthermore, the sites 0 and $2 R+2$ are updated with rates proportional to $\lambda$. The interesting case, from the modeling perspective, is that in which $\lambda$ is much smaller than one: namely, the boundary sites are slowed down and mimic the action of large particle reservoirs.

\section{UPHILL CURRENTS IN THE ZRP-OB}

In this section we shall prove that the ZRP-OB can exhibit stationary uphill currents. More precisely, we shall consider 
the process described by the generator given in (4) and show that, for a particular choice of the parameters, the steady state is characterized by a current flowing from the reservoir with smaller density to the one with larger density.

\section{A. Stationary measure for the ZRP-OB}

Consider the ZRP-OB defined in Sec. II A. A probability measure $\mu_{R}$ on $\Omega_{R}$ is stationary for the ZRP if and only if

$$
\sum_{n \in \Omega_{R}} \mu_{R}(n)\left(L_{R} f\right)(n)=0
$$

for any function $f$. A sufficient condition is provided by the balance equation

$$
\sum_{m \neq n} \mu_{R}(m) r(m, n)=\mu_{R}(n) \sum_{m \neq n} r(n, m)
$$

for any $n \in \Omega_{R}$.

Consider the positive reals $s_{1}, \ldots, s_{2 R+1}$, called fugacities, and the product measure on the space $\Omega_{R}$ defined as

$$
v_{R}(n)=\prod_{x=1}^{2 R+1} v_{x}\left(n_{x}\right) \quad \text { with } \quad v_{x}\left(n_{x}\right)=\frac{1}{Z_{x}} \frac{s_{x}^{n_{x}}}{u_{x}\left(n_{x}\right) !},
$$

where $u_{x}(k) !=1$ if $k=0$ and $u_{x}(k) !=u_{x}(1) \cdots u_{x}(k)$ if $k \geqslant$ 1 and

$$
Z_{x}=\sum_{k=0}^{\infty} \frac{s_{x}^{k}}{u_{x}(k) !}
$$

By exploiting Eq. (11), or by applying (10) to the functions $f(n)=n_{x}$ for any $x$, it can be proven that $v$ is stationary for the ZRP-OB provided the reals $s_{x}$ satisfy the following equations:

$$
\begin{aligned}
(\gamma+p) s_{1} & =\alpha+q s_{2}, \\
(q+p) s_{x} & =p s_{x-1}+q s_{x+1} \quad \text { for } x=2, \ldots, R-1, \\
(q+p) s_{R} & =p s_{R-1}+\bar{q} s_{R+1}, \\
(\bar{q}+\bar{p}) s_{R+1} & =p s_{R}+q s_{R+2}, \\
(q+p) s_{R+2} & =\bar{p} s_{R+1}+q s_{R+3}, \\
(q+p) s_{x} & =p s_{x-1}+q s_{x+1} \quad \text { for } x=R+3, \ldots, 2 R \\
(q+\beta) s_{2 R+1} & =p s_{2 R}+\delta .
\end{aligned}
$$

After some simple algebra we get the equations

$$
\begin{aligned}
p s_{R}-\bar{q} s_{R+1} & =p s_{R-1}-q s_{R}, \\
\bar{p} s_{R+1}-q s_{R+2} & =p s_{R}-\bar{q} s_{R+1}, \\
p s_{2 R}-q s_{2 R+1} & =\beta s_{2 R+1}-\delta, \\
p s_{x}-q s_{x+1} & =\alpha-\gamma s_{1}
\end{aligned}
$$

for $x=1, \ldots, R-1$ and $x=R+2, \ldots, 2 R$, which reduce to Eq. (30) in Ref. [30] in the case $(\bar{q}, \bar{p})=(q, p)$. These equations admits a unique solution to be discussed in detail in the sequel for a particular choice of the parameters $p, q, \bar{p}, \bar{q}, \alpha, \beta, \gamma$, and $\delta$.

\section{B. Stationary current and density profile for the ZRP-OB}

The main quantities of interest, in our study, are the stationary density or occupation number profiles

$$
\rho_{x}=v_{x}\left[n_{x}\right]=\frac{1}{Z_{x}} \sum_{k=1}^{\infty} k \frac{s_{x}^{k}}{u(k) !}=s_{x} \frac{\partial}{\partial s_{x}} \log Z_{x}
$$

(see Ref. [31] for the details) and the stationary current

$$
\begin{aligned}
J_{R, x} & =v_{R}\left[u\left(n_{x}\right) p_{x}-u\left(n_{x+1}\right) q_{x+1}\right] \\
& =p_{x} v_{x}\left[u\left(n_{x}\right)\right]-q_{x+1} v_{x+1}\left[u\left(n_{x+1}\right)\right] \\
& =p_{x} s_{x}-q_{x+1} s_{x+1}
\end{aligned}
$$

for $x=1, \ldots, 2 R$, where we omitted the last straightforward computation. The stationary current represents the difference between the average number of particles crossing a bond between two adjacent sites on the lattice from the left to the right and the corresponding number in the opposite direction. Equations (15) shows that the stationary current does not depend on the site $x$, therefore we shall simply write $J_{R} \equiv J_{R, x}$.

Note that it was possible to express the current in terms of the fugacities without relying on any specific choice for the intensity function $u$. Yet an explicit form for $u$ is needed in the computation of the density profile. In general it can be proven (see Ref. [31]) that

$$
\frac{\partial \rho_{x}}{\partial s_{x}}=\frac{1}{s_{x}}\left\{v_{x}\left[n_{x}^{2}\right]-\left(v_{x}\left[n_{x}\right]\right)^{2}\right\}>0,
$$

hence, at each site, the stationary mean occupation number is an increasing function of the local fugacity.

Particularly relevant cases are the so-called independent particle and the simple exclusion-like ZRP models, in which the intensity function is respectively given by $u(k)=k$ and $u(k)=1$ for $k \geqslant 1$ [recall that $u(0)=0$ ]. In these two cases it is easy to prove that $Z_{x}^{\mathrm{ip}}=\exp \left\{s_{x}\right\}$ and $Z_{x}^{\mathrm{se}}=1 /\left(1-s_{x}\right)$ for $s_{x}<1$, respectively. Hence, by (16), one has

$$
\rho_{x}^{\mathrm{ip}}=s_{x} \quad \text { and } \quad \rho_{x}^{\mathrm{se}}=\frac{s_{x}}{1-s_{x}} \text { for } s_{x}<1
$$

for the independent particle and the simple exclusion-like models, respectively.

\section{The almost everywhere symmetric ZRP-OB}

We shall fix $q=p=\gamma=\beta=1 / 2, \bar{q}=1 / 2-\epsilon$, and $\bar{p}=$ $1 / 2+\epsilon$ for some $\epsilon \in[0,1 / 2)$. In this case, the solution of Eqs. (15) is linear in $x$ and can be written as

$$
s_{x}= \begin{cases}\sigma x+2 \alpha & \text { for } x=1, \ldots, R \\ \alpha+\delta & \text { for } x=R+1 \\ \sigma x+2 \alpha+4 \epsilon(\alpha+\delta) & \text { for } x=R+2, \ldots, 2 R+1\end{cases}
$$

with slope

$$
\sigma=\frac{1}{R+1}[\delta-\alpha-2 \epsilon(\alpha+\delta)]
$$



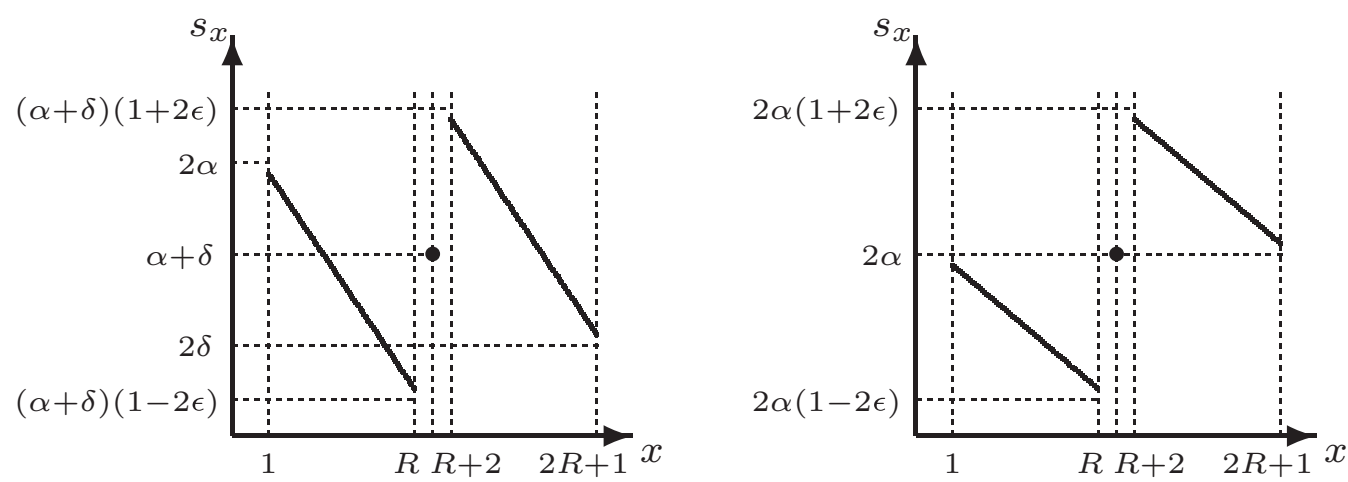

FIG. 2. Fugacity profile, for $R$ large, in the case $\alpha>\delta$ (left panel) and $\alpha=\delta$ (right panel).

To draw the fugacity profiles $s_{x}$ for $x=1, \ldots, 2 R+1$, shown in Figs. 2 and 3, it is useful to compute

$$
\begin{aligned}
s_{1} & =\sigma+2 \alpha, \\
s_{R} & =-\sigma+(\alpha+\delta)(1-2 \epsilon), \\
s_{R+2} & =\sigma+(\alpha+\delta)(1+2 \epsilon), \\
s_{2 R+1} & =-\sigma+2 \delta .
\end{aligned}
$$

In the case $\alpha>\delta$, recalling that $\epsilon \in[0,1 / 2)$, we have that $\sigma<0,2 \alpha>\alpha+\delta>2 \delta, 0<(\alpha+\delta)(1-2 \epsilon) \leqslant \alpha+\delta$, and $\alpha+\delta \leqslant(\alpha+\delta)(1+2 \epsilon)<2(\alpha+\delta)$. This explains the graph shown in the left panel of Fig. 2, portraying the fugacity profiles for $R$ large, in which case the terms $\pm \sigma$ in (22) are small.

In the case $\alpha=\delta$, recalling that $\epsilon \in[0,1 / 2)$, we have that $\sigma=-4 \epsilon \alpha /(R+1)<0$ and $0<2 \alpha(1-2 \epsilon) \leqslant 2 \alpha \leqslant$ $2 \alpha(1+2 \epsilon)$. This case is shown in the right panel of Fig. 2, where the terms $\pm \sigma$ are still assumed to be small.

The discussion of the the case $\alpha<\delta$ is more delicate, since the sign of $\sigma$ depends on the value of the difference $\delta-\alpha$. More precisely, it holds

$$
\sigma \geqslant 0 \text { if and only if } \epsilon \leqslant \frac{1}{2} \frac{\delta-\alpha}{\alpha+\delta} \equiv \epsilon_{\mathrm{c}},
$$

where we have introduced the critical bias $\epsilon_{\mathrm{c}}$. Thus, we have to distinguish three cases.

For $0 \leqslant \epsilon<\epsilon_{\mathrm{c}}$ we have that $\sigma>0,2 \alpha<(\alpha+\delta)(1-$ $2 \epsilon) \leqslant \alpha+\delta$, and $\alpha+\delta \leqslant(\alpha+\delta)(1+2 \epsilon)<2 \delta$. For $\epsilon=\epsilon_{\mathrm{c}}$ we have that $\sigma=0,(\alpha+\delta)\left(1-2 \epsilon_{\mathrm{c}}\right)=2 \alpha$, and $(\alpha+\delta)(1+$ $\left.2 \epsilon_{\mathrm{c}}\right)=2 \delta$. For $\epsilon_{\mathrm{c}}<\epsilon<1 / 2$ we have that $\sigma<0,2 \alpha>$ $(\alpha+\delta)(1-2 \epsilon)>0$, and $2(\alpha+\delta)>(\alpha+\delta)(1+2 \epsilon)>2 \delta$.
The graphs in Fig. 3 represent the fugacity profiles for $R$ large, in the three cases.

Remarkably, the presence of a critical value for the bias, marking the transition from a regime of standard (downhill) diffusion to another regimes of uphill diffusion, was also reported in Ref. [15]; cf. Figure 4 therein. In that work, much in the same spirit of the ZRP-OB model, the diffusion of particles in the channel results from the balance between the standard diffusive behavior induced by the reservoirs and the uphill motion triggered by the Kac potential in the bulk (whose effect is only visible in a neighborhood around the central site of the lattice).

As already mentioned in Sec. III B, the stationary current can be computed from the knowledge of the fugacity profile without specifying the intensity function. Applying (17) and (20) we find

$$
J_{R}=-\frac{1}{2} \sigma=-\frac{1}{2(R+1)}[\delta-\alpha-2 \epsilon(\alpha+\delta)] .
$$

On the other hand, to compute the density profile it is necessary to consider a particular form for the intensity function; see (19) for the independent particle and the simple exclusion-like cases.

For the independent particle model, the fugacity profiles shown in Figs. 2 and 3 correspond to the density profiles. In particular, by summing up the density profile $\rho_{x}$ for $x=$ $1, \ldots, 2 R+1$, we find the average total number of particles in the channel in the steady state:

$$
N^{\text {ip }}=(\alpha+\delta)(2 R+1) .
$$
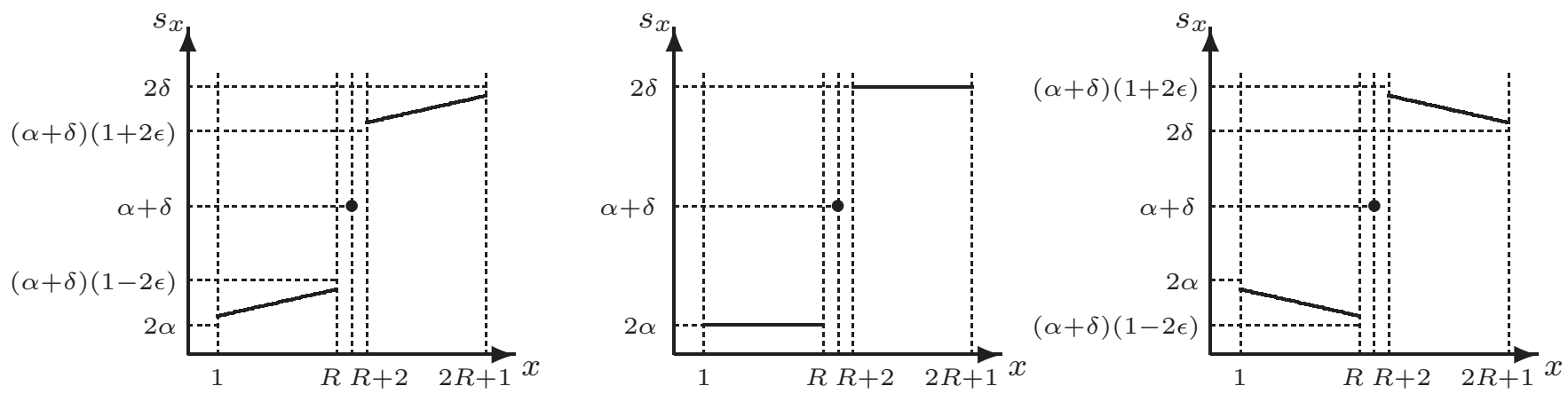

FIG. 3. Fugacity profile, for $R$ large, in the case $\alpha<\delta$ for $\epsilon<\epsilon_{\mathrm{c}}$ (left panel), $\epsilon=\epsilon_{\mathrm{c}}$ (central panel), and $\epsilon_{\mathrm{c}}<\epsilon$ (right panel). 

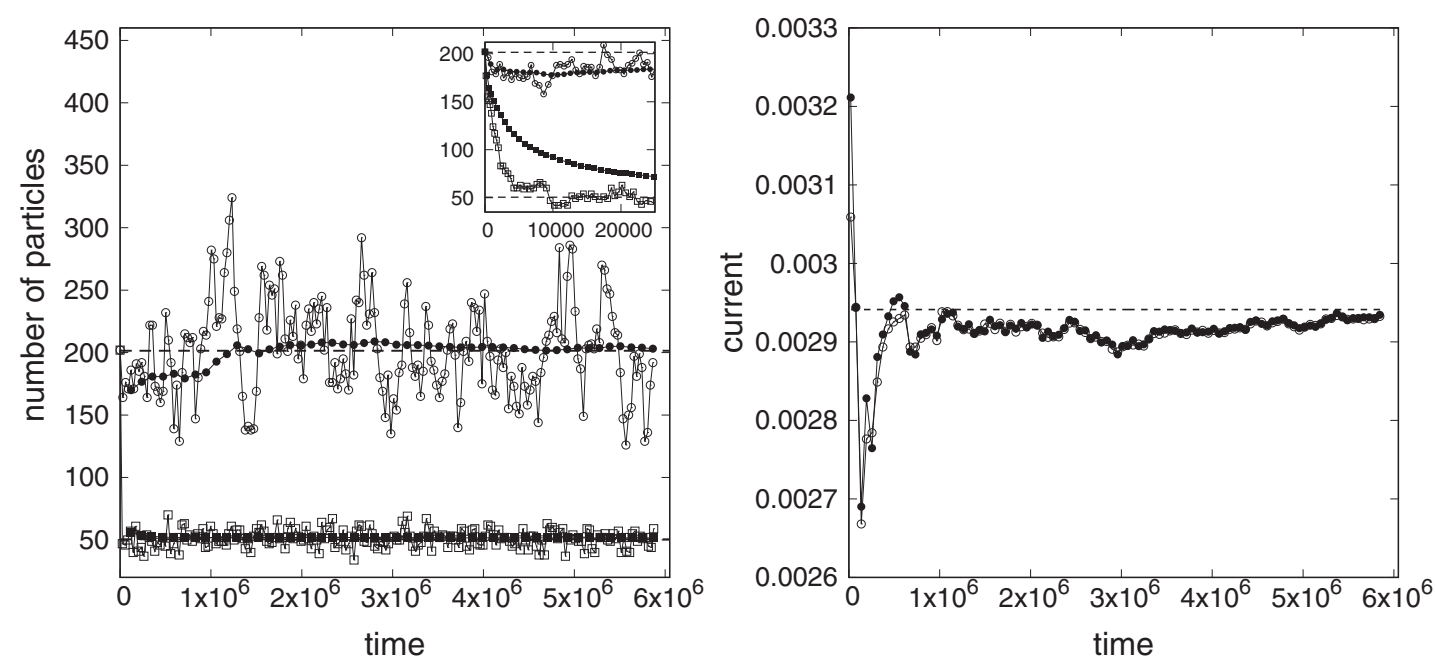

FIG. 4. Monte Carlo results for the almost everywhere symmetric ZRP-OB with $R=50, \epsilon=0.4, \alpha=0.2, \delta=0.3$. The initial datum used in the simulations is a uniform configuration with two particles per site. Squares and circles refer, respectively, to the independent particle and the simple exclusion-like models. The dashed lines represent the exact solution. Left panel: the total number of particles in the system is measured vs time. The open symbols are the instantaneous values, and the solid symbols are the time-averaged values. Time is measured from the beginning of the dynamics. The inset in the upper right corner is a magnification of the larger figure at short times and shows the rapid relaxation of the time-averaged total number of particles in the independent particle case to the theoretical value given in (25). Right panel: particle currents measured at the boundaries vs time. Open and solid symbols refer, respectively, to the left and the right boundary. Time is counted starting from the "thermalization" time $2 \times 10^{6}$, taken as the origin of the horizontal axis.

Moreover, in the case $\alpha>\delta, \sigma<0$ implies that $J_{R}>0$. The current goes downhill, i.e., it flows from the reservoir with larger density (characterized by the injection rate $\alpha$ ) towards the reservoir with smaller density (with injection rate $\delta$ ).

When $\alpha=\delta, \sigma<0$ implies that $J_{R}>0$. The diffusion is now uphill: indeed, in spite of the equality of the injection rates, the current goes from the boundary site 1 , with lower density, to the site $2 R+1$, with higher density. The effect, though, is barely visible because $s_{2 R+1}-s_{1}=8 \epsilon \alpha /(R+1)$ vanishes for $R$ large. It is also interesting to note that, for $R$ sufficiently large, the density profile corresponding to the case $\alpha=\delta$ recovers, qualitatively, the plot portrayed in Fig. 2 of Ref. [15], referring to a scenario similar to the one considered here for the ZRP-OB model, where the injection rates at the boundaries coincide.

In the case $\alpha<\delta$, finally, for $\epsilon<\epsilon_{\mathrm{c}}$ the diffusion is downhill, for $\epsilon=\epsilon_{\mathrm{c}}$ the current vanishes, and for $\epsilon_{\mathrm{c}}<\epsilon$ the diffusion is uphill.

We cannot write a general formula for the density profile for any choice of the intensity function $u$. But, using (16), we have that $\rho_{x+1}>\rho_{x}$ if and only if $s_{x+1}>s_{x}$. This implies that the results we deduced for the independent particle model are, indeed, completely general.

Let us now compare our exact results with the Monte Carlo simulations. The model has been simulated as follows: call $n$ the configuration at time $t$, then (i) a number $\tau$ is picked up at random with exponential distribution of parameter $U=$ $\alpha+\delta+\sum_{x=1}^{2 R+1} u_{x}\left(n_{x}\right)$ and time is updated to $t+\tau$; (ii) an integer $y$ in $0,1, \ldots, 2 R+2$ is chosen at random on the lattice with probability $\pi_{y}=u_{y}\left(n_{y}\right) / U$ for $y=1, \ldots, 2 R+1, \pi_{0}=$ $\alpha / U$, and $\pi_{2 R+2}=\delta / U$ (note that, for simplicity, we skipped the time dependence in the notation); (iii) if $y \neq 0,2 R+2$ a particle is moved from the site $y$ to the site $y+1$ with probability $p_{y} /\left(q_{y}+p_{y}\right)$ (in the case $y=2 R+1$ the particle is removed) or to the site $y-1$ with probability $q_{y} /\left(q_{y}+p_{y}\right)$ (in the case $y=1$ the particle is removed), if $y=0$ a particle is added to the site 1 , if $y=2 R+2$ a particle is added to the site $2 R+1$.

In Fig. 4 we report the Monte Carlo measure of the total number of particles in the channel and of the boundary currents as functions of time, for the independent particle and the simple exclusion-like models. The values of the parameters used in the simulations are indicated in the caption. Both panels of Fig. 4 show that when time is large enough the time-averaged values of the total number of particles and of the currents tend to the analytical results (24) and (25) (dashed lines). Concerning the theoretical value of the total number of particles, note that the analytic expression (25) applies only to the independent particle case; in the simple exclusion-like model we summed up numerically, for $x=1, \ldots, 2 R+1$, the values of $\rho_{x}$ given by (19), with $s_{x}$ in (20).

The data in Fig. 4 (left panel) also give an insight into the magnitude of the "thermalization" time, namely, the time interval in which the time-averaged total number of particles converges to the corresponding theoretical stationary value. As visible in the inset of Fig. 4 (left panel), the thermalization time in the independent particle case is considerably smaller than that observed in the simple exclusion-like model, which is of the order of $2 \times 10^{6}$ (for the given initial datum used in the simulations). It should also be noted that the steady state fluctuations of the instantaneous total number of particles around the time-averaged value are larger in the simple exclusion-like model. To numerically check the convergence of the current to its theoretical value [see Fig. 4 (right panel)], we thus skipped the initial transient dynamics and measured the current starting from the time $2 \times 10^{6}$. 

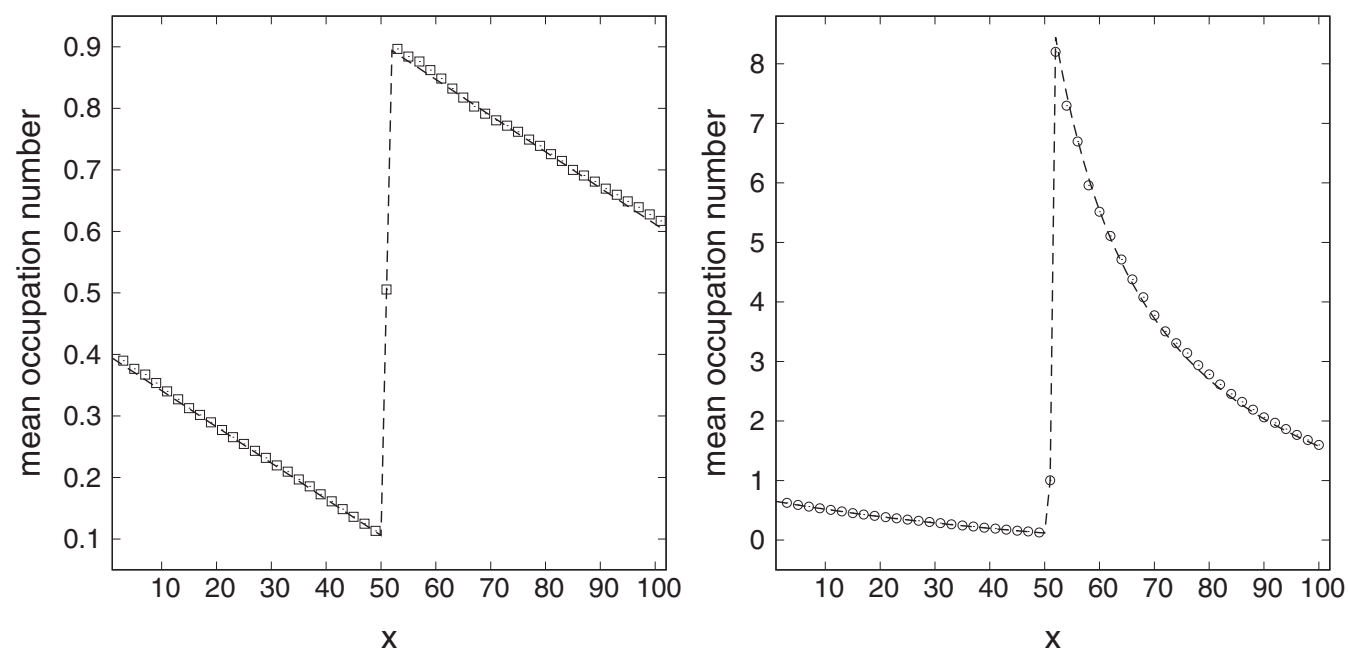

FIG. 5. Monte Carlo results for the density profile of the almost everywhere symmetric ZRP-OB with $R=50, \epsilon=0.4, \alpha=0.2$, and $\delta=0.3$. Squares and circles refer, respectively, to the independent particle (left panel) and the simple exclusion-like (right panel) models. The dashed lines represent the exact solution given in (19). The stationary density profiles have been computed by averaging, after the thermalization time is reached, over a set of $10^{6}$ instantaneous particle configurations.

The same procedure was also adopted for the measure of the stationary density profiles, reported in Fig. 5. The match between the Monte Carlo numerical measure and the exact results is striking. As expected [see (19)], the density profile in the simple exclusion-like model is not linear. Note also that in the simple exclusion-like model no symmetry between the left and the right halves of the lattice exists. Moreover, though the values of the boundary rates $\alpha$ and $\delta$ used in the simulations are the same in the two considered models, completely different values of the density at the boundary sites are obtained. This suggests, hence, that the dynamics in the bulk significantly affects the value of the density at the boundaries.

\section{UPHILL CURRENTS IN THE ZRP-CC}

In this section we shall also prove that the ZRP-CC can exhibit anomalous uphill currents. More precisely, we shall consider the process described by the generator given in (9) and discuss the effect produced, in the steady state, by the local asymmetry in the bulk and by the two slow boundary sites.

\section{A. Stationary measure for the ZRP-CC}

For the periodic ZRP introduced in Sec. II B, the invariant measure $\mu_{R, N}$ satisfies

$$
\sum_{n \in \Omega_{R, N}} \mu_{R, N}(n)\left(L_{R, N} f\right)(n)=0
$$

With arguments similar to those developed in Sec. III [see also Eq. (15) in Ref. [29]], it can be proven that the invariant or stationary measure of the ZRP-CC process attains the form

$$
v_{R, N}(n)=\frac{1}{Z_{R, N}} \prod_{x=0}^{2 R+2} \frac{s_{x}^{n_{x}}}{u\left(n_{x}\right) !}
$$

for any $n \in \Omega_{R, N}$, where the partition function $Z_{R, N}$ is the normalization constant

$$
Z_{R, N}=\sum_{n \in \Omega_{R, N}} \prod_{x=0}^{2 R+2} \frac{s_{x}^{n_{x}}}{u\left(n_{x}\right) !}
$$

and $s_{0}, \ldots, s_{2 R+2}$ are not negative real numbers satisfying the following equations:

$$
\begin{aligned}
2 \lambda s_{0} & =q s_{1}+\lambda s_{2 R+2} \\
(q+p) s_{1} & =\lambda s_{0}+q s_{2} \\
(q+p) s_{x} & =p s_{x-1}+q s_{x+1} \quad \text { for } x=2, \ldots, R-1 \\
(q+p) s_{R} & =p s_{R-1}+\bar{q} s_{R+1} \\
(\bar{q}+\bar{p}) s_{R+1} & =p s_{R}+q s_{R+2}, \\
(q+p) s_{R+2} & =\bar{p} s_{R+1}+q s_{R+3} \\
(q+p) s_{x} & =p s_{x-1}+q s_{x+1} \quad \text { for } x=R+3, \ldots, 2 R \\
(q+p) s_{2 R+1} & =p s_{2 R}+\lambda s_{2 R+2} .
\end{aligned}
$$

With simple algebra we get the equations

$$
\begin{aligned}
2 \lambda s_{0} & =q s_{1}+\lambda s_{2 R+2}, \\
p s_{R}-\bar{q} s_{R+1} & =p s_{R-1}-q s_{R}, \\
\bar{p} s_{R+1}-q s_{R+2} & =p s_{R}-\bar{q} s_{R+1}, \\
p s_{2 R+1}-\lambda s_{2 R+2} & =p s_{2 R}-q s_{2 R+1}, \\
p s_{x}-q s_{x+1} & =\lambda s_{0}-q s_{1}
\end{aligned}
$$

for $x=1, \ldots, R-1$ and $x=R+2, \ldots, 2 R$. These equations admits a class of $\infty^{1}$ solutions that will be discussed in detail in the sequel for a particular choice of the parameters $p, q, \bar{p}, \bar{q}$, and $\lambda$ defining the rates. 

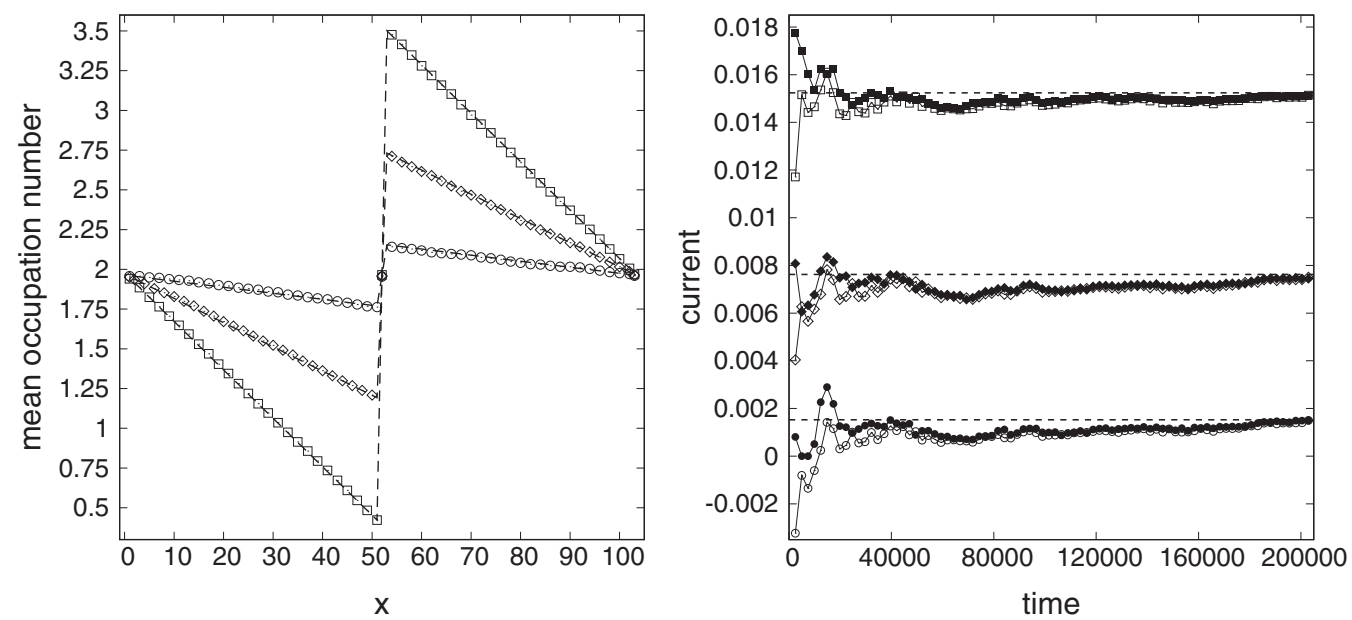

FIG. 6. Results for the almost everywhere symmetric ZRP-BB model with $R=50, N=206, \lambda=0.25, \epsilon=0.05$ (circles), 0.2 (diamonds), 0.4 (squares). Left panel: stationary density profiles, obtained as discussed in Fig. 5. Open symbols denote the Monte Carlo measures and the dashed lines the exact solution in (37) and (38). Note that the average occupation numbers of the finite reservoirs in $x=0$ and in $x=2 R+2$ have been multiplied by the factor $\lambda$ to fit within the figure. Right panel: particle currents measured on the bonds $(2 R+1)-(2 R+2)($ open symbols) and $(2 R+2)-(0)$ (solid symbols). The dashed lines represent the theoretical prediction in (41). Time, on the horizontal axis, is counted from the "thermalization" time $2 \times 10^{6}$, as in Fig. 4 .

\section{B. Stationary current and density profile for the ZRP-CC}

We shall focus, again, on the stationary density profile

$$
\rho_{R, N, x}=v_{R, N}\left[n_{x}\right]=\frac{1}{Z_{R, N}} \sum_{n \in \Omega_{R, N}} n_{x} \prod_{y=0}^{2 R+2} \frac{s_{x}^{n_{y}}}{u\left(n_{y}\right) !}
$$

and the stationary current

$$
J_{R, N, x}=v_{R, N}\left[u\left(n_{x}\right) p_{x}-u\left(n_{x+1}\right) q_{x+1}\right]
$$

for $x=1, \ldots, 2 R$. With the same arguments used to prove Eq. (11) in Ref. [29] we get

$$
v_{R, N}\left[u\left(n_{x}\right)\right]=\frac{Z_{R, N-1}}{Z_{R, N}} s_{x},
$$

hence

$$
J_{R, N, x}=\frac{Z_{R, N-1}}{Z_{R, N}}\left(p_{x} s_{x}-q_{x+1} s_{x+1}\right)
$$

for $x=1, \ldots, 2 R$, where the last equality follows from Eq. (11) in Ref. [29]. Equations (30) prove that the current does not depend on the site $x$, hence we shall simply write $J_{R, N} \equiv J_{R, N, x}$.

In this periodic case it is not possible to push forward the discussion without embracing a specific form for the intensity function. Thus, from now onwards in this section, we shall restrict our description to the independent particle case $u(k)=$ $k$ and add the superscript "ip" to the notation. We first compute the partition function

$$
Z_{R, N}^{\mathrm{ip}}=\sum_{n \in \Omega_{R, N}} \prod_{x=0}^{2 R+2} \frac{s_{x}^{n_{x}}}{n_{x} !}=\frac{1}{N !}\left[\sum_{x=0}^{2 R+2} s_{x}\right]^{N},
$$

where we used the convention $0 !=1$ and applied the multinomial theorem [Eq. (3.35) in Ref. [32]]. From (34) (and from the notational remark below it) we then get

$$
J_{R, N}^{\mathrm{ip}}=N\left[\sum_{x=0}^{2 R+2} s_{x}\right]^{-1}\left(p_{x} s_{x}-q_{x+1} s_{x+1}\right) .
$$

Moreover, since $u\left(n_{x}\right)=n_{x}$, Eq. (33) can be also used to compute the density profile:

$$
\rho_{x}^{\text {ip }}=v_{R, N}^{\text {ip }}\left[n_{x}\right]=v_{R, N}^{\text {ip }}\left[u\left(n_{x}\right)\right]=N\left[\sum_{x=0}^{2 R+2} s_{x}\right]^{-1} s_{x},
$$

where in the last step we used (33) and (35). Note that $\rho_{0}^{\text {ip }}$ and $\rho_{2 R+2}^{\text {ip }}$ correspond to the average total number of particles allocated, respectively, in the left and in the right reservoirs. Retaining the interpretation discussed at the end of Sec. II A, from the expression of the injection rates given in (5) and (6) we find that the average particle densities in the two reservoirs take the values, respectively, $\lambda \rho_{0}^{\text {ip }}$ and $\lambda \rho_{2 R+2}^{\text {ip }}$. Thus, in the ZRP-CC model, the two slow sites act as finite particle reservoirs, each constituted by $\lambda^{-1}$ sites. In Fig. 6 (right panel) are shown, hence, the density profile in the bulk, i.e., for $x=1, \ldots, 2 R+$ 1 , and at the slow sites in $x=0$ and in $x=2 R+2$.

\section{The almost everywhere symmetric ZRP-CC}

Let us fix $q=p=\gamma=\beta=1 / 2, \bar{q}=1 / 2-\epsilon$, and $\bar{p}=$ $1 / 2+\epsilon$ for some $\epsilon \in[0,1 / 2)$. In this case the solution of 
Eqs. (30) is linear in $x$ and can be written as

$$
s_{x}= \begin{cases}\sigma x+2 \lambda s_{0} & \text { for } x=1, \ldots, R \\ 2 \lambda(3+2 R) s_{0} /(3+2 R-2 \epsilon) & \text { for } x=R+1 \\ \sigma x+2 \lambda\left[1+4 \epsilon+8 \epsilon^{2} /(3+2 R-2 \epsilon)\right] s_{0} & \text { for } x=R+2, \ldots, 2 R+1 \\ {[1+4 \epsilon /(3+2 R-2 \epsilon)] s_{0}} & \text { for } x=2 R+2\end{cases}
$$

with slope

$$
\sigma=-\frac{8 \lambda \epsilon s_{0}}{3+2 R-2 \epsilon}
$$

and $s_{0}$ arbitrary. Moreover, we have that

$$
\sum_{x=0}^{2 R+2} s_{x}=\frac{2(3+2 R)[1+\lambda(2 R+1)]}{3+2 R-2 \epsilon} s_{0} .
$$

Hence, (36) and (37) yield

$$
J_{R, N}^{\mathrm{ip}}=-\frac{1}{2} \sigma N\left[\sum_{x=0}^{2 R+2} s_{x}\right]^{-1}=\frac{2 \lambda \epsilon N}{(3+2 R)[1+\lambda(2 R+1)]}
$$

$$
\rho_{0}^{\text {ip }}=\frac{N(3+2 R-2 \epsilon)}{2(3+2 R)[1+\lambda(2 R+1)]},
$$

and

$$
\rho_{2 R+2}^{\mathrm{ip}}=\frac{N(3+2 R+2 \epsilon)}{2(3+2 R)[1+\lambda(2 R+1)]} .
$$

In conclusion,

$$
\rho_{2 R+2}^{\text {ip }}-\rho_{0}^{\text {ip }}=\frac{2 \epsilon N}{(3+2 R)[1+\lambda(2 R+1)]}>0
$$

and $J_{R, N}^{\mathrm{ip}}>0$, which proves that the channel is crossed by an uphill current flowing from the reservoir with lower particle density (in $x=0$ ) to the one with higher particle density (in $x=2 R+2)$.

We have numerically simulated the almost everywhere symmetric ZRP-CC model following a scheme similar to that outlined in Sec. III C. We find, also in this case, an optimal match between the exact density profiles obtained from (37) and (38) and the numerical data; see Fig. 6.

\section{THE HYDRODYNAMIC LIMIT}

We discuss on heuristic grounds the hydrodynamic limit $[28,33]$ of the almost everywhere symmetric ZRP-OB model introduced in Sec. III C, with the intensity function corresponding to the independent particle case, namely, $u(k)=k$.

For any $i \in \Lambda$ set $x_{i}=i /(2 R+1)$ so that $x_{i} \in[1 /(2 R+$ $1), 1]$. Denote by $n_{i}(t)$ the time-dependent density profile at time $t$, i.e., $n_{i}(t)$ is the average number of particles occupying the site $i$ at time $t$. The change of the number of particles at a site in the bulk, i.e., $i \neq 1, R, R+1, R+2,2 R+1$, in a small interval $\Delta t$, can be estimated as

$$
n_{i}(t+\Delta t)-n_{i}(t)=-n_{i}(t) \Delta t+\frac{1}{2} n_{i-1}(t) \Delta t+\frac{1}{2} n_{i+1}(t) \Delta t .
$$

This equality can be rewritten as

$$
\frac{n_{i}(t+\Delta t)-n_{i}(t)}{\Delta t /(2 R+1)^{2}}=\frac{\left[n_{i+1}(t)-n_{i}(t)\right]-\left[n_{i}(t)-n_{i-1}(t)\right]}{2 /(2 R+1)^{2}} .
$$

Thus, if time is rescaled as $t /(2 R+1)^{2} \rightarrow t$ (diffusive scaling), in the limit $R \rightarrow \infty$ the particle density profile $n_{i}(t)$ will tend to a function $u(x, t)$ solving the diffusion equation

$$
\frac{\partial u}{\partial t}=\frac{1}{2} \frac{\partial^{2} u}{\partial x^{2}} \quad \text { in }(0,1 / 2) \cup(1 / 2,1) .
$$

In order to guess the boundary conditions at $x=0,1 / 2,1$ we shall write the balance equation of the currents at the sites $x_{1}, x_{R}, x_{R+1}, x_{R+2}$, and $x_{2 R+1}$. More precisely, we consider a small interval of time $\Delta t$, and we first write

$$
\alpha \Delta t-n_{1}(t) \Delta t+\frac{1}{2} n_{2}(t) \Delta t=0
$$

and

$$
\delta \Delta t-n_{2 R+1}(t) \Delta t+\frac{1}{2} n_{2 R}(t) \Delta t=0,
$$

which, in the limit $R \rightarrow \infty$, provide the boundary conditions

$$
u(0, t)=2 \alpha \quad \text { and } \quad u(1, t)=2 \delta .
$$

Note that Eqs. (43) are obtained by assuming that the injection rates $\alpha$ and $\delta$ are independent of $R$; different boundary conditions may hold under different scalings of $\alpha$ and $\delta$ with $R$. Moreover, we have that

$$
\begin{aligned}
& \frac{1}{2} n_{R-1}(t) \Delta t-n_{R}(t) \Delta t+\left(\frac{1}{2}-\epsilon\right) n_{R+1}(t) \Delta t=0, \\
& \frac{1}{2} n_{R}(t) \Delta t-n_{R+1}(t) \Delta t+\frac{1}{2} n_{R+2}(t) \Delta t=0, \\
& \left(\frac{1}{2}+\epsilon\right) n_{R+1}(t) \Delta t-n_{R+2}(t) \Delta t+\frac{1}{2} n_{R+3}(t) \Delta t=0 .
\end{aligned}
$$

The equation in the middle can be rewritten as

$$
\frac{1}{2}\left[n_{R}(t)-n_{R+1}(t)\right]=\frac{1}{2}\left[n_{R+1}(t)-n_{R+2}(t)\right],
$$

which, divided by $1 /(2 R+1)$, in the limit $R \rightarrow \infty$ provides the condition

$$
\lim _{x \rightarrow 1 / 2^{-}} \frac{\partial}{\partial x} u(x, t)=\lim _{x \rightarrow 1 / 2^{+}} \frac{\partial}{\partial x} u(x, t) .
$$

Combining the first and the third equation, on the other hand, we get

$$
\begin{aligned}
& {\left[\frac{1}{2} n_{R-1}(t)-n_{R}(t)\right]\left(\frac{1}{2}+\epsilon\right)} \\
& \quad+\left[n_{R+2}(t)-\frac{1}{2} n_{R+3}(t)\right]\left(\frac{1}{2}-\epsilon\right)=0 .
\end{aligned}
$$

Since in the limit $R \rightarrow \infty$ we have that $\left[n_{R-1}(t)-n_{R}(t)\right] / 2$ and $\left[n_{R+2}(t)-n_{R+3}(t)\right] / 2$ tend to zero, the above equation can be interpreted as

$$
\left(\frac{1}{2}+\epsilon\right) \lim _{x \rightarrow 1 / 2^{-}} u(x, t)=\left(\frac{1}{2}-\epsilon\right) \lim _{x \rightarrow 1 / 2^{+}} u(x, t) .
$$


In conclusion, we find that the evolution of the model in the hydrodynamic limit is described by the differential equation (42) supplemented with the boundary conditions (43), (44), and (45). In particular, the stationary profile is the solution of the problem

$$
\begin{aligned}
u^{\prime \prime}(x) & =0 \\
u(0) & =2 \alpha \text { and } u(1)=2 \delta \\
u_{-}^{\prime}(1 / 2) & =u_{+}^{\prime}(1 / 2) \\
(1 / 2+\epsilon) u_{-}(1 / 2) & =(1 / 2-\epsilon) u_{+}(1 / 2),
\end{aligned}
$$

where the subscripts - and + denote, respectively, the left and the right limits.

The stationary problem (46) can be easily solved: one can write $u(x)=A x+B$ for $x \in(0,1 / 2)$ and $u(x)=C x+D$ for $x \in(1 / 2,1)$. The boundary conditions then yield

$$
u(x)=2[\delta-\alpha-2 \epsilon(\alpha+\delta)] x+2 \alpha
$$

for $0 \leqslant x \leqslant 1 / 2$ and

$$
u(x)=2[\delta-\alpha-2 \epsilon(\alpha+\delta)] x+2 \alpha+4 \epsilon(\alpha+\delta)
$$

for $1 / 2 \leqslant x \leqslant 1$. The solution of the macroscopic stationary equation matches perfectly with the stationary density profile of the microscopic lattice model. Indeed, by performing the change of variable $x /(2 R+1) \rightarrow x$ in (20), one finds, for $R$ large, Eqs. (47) and (48).

It is also possible to solve the time dependent problem (42)-(45) and write the solution in terms of a Fourier series. We first introduce the functions

$$
Y_{1}(x, t)=u(x, t) \text { and } Y_{2}(x, t)=u(1-x, t)
$$

for $x \in[1,1 / 2]$ and note that the conditions (43)-(45) imply

$$
Y_{1}(0, t)=2 \alpha, Y_{2}(0, t)=2 \delta, \frac{\partial Y_{1}}{\partial x}\left(\frac{1}{2}, t\right)+\frac{\partial Y_{2}}{\partial x}\left(\frac{1}{2}, t\right)=0
$$

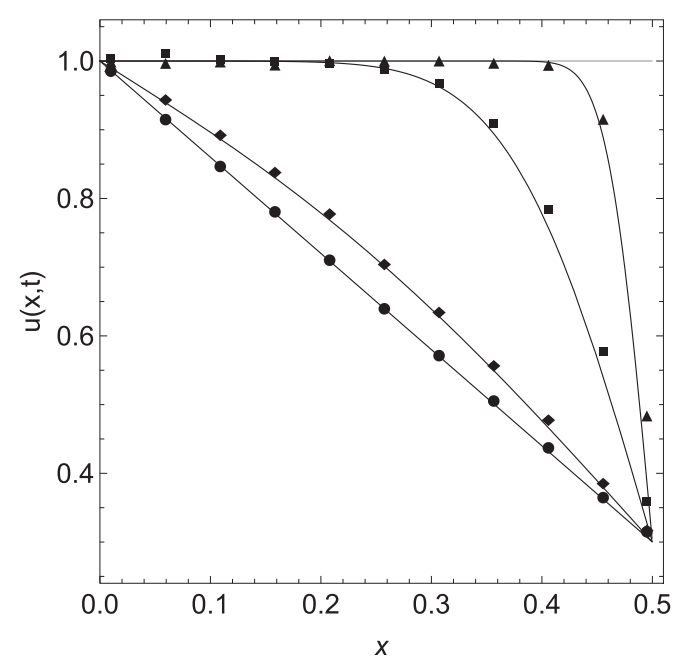

and

$$
\left(\frac{1}{2}+\epsilon\right) Y_{1}(1 / 2, t)-\left(\frac{1}{2}-\epsilon\right) Y_{2}(1 / 2, t)=0 .
$$

Moreover, by (42) we have that both $Y_{1}$ and $Y_{2}$ solve the heat equation with diffusion coefficient $1 / 2$. As a second step, we introduce the functions

$$
W(x, t)=Y_{1}(x, t)+Y_{2}(x, t)
$$

and

$$
U(x, t)=\left(\frac{1}{2}+\epsilon\right) Y_{1}(x, t)-\left(\frac{1}{2}-\epsilon\right) Y_{2}(x, t),
$$

and note that from the boundary conditions on $Y_{1}$ and $Y_{2}$ we get

$$
W(0, t)=2(\alpha+\delta), W(1 / 2, t)=2(\alpha+\delta)
$$

and

$$
U(0, t)=\alpha-\delta+2 \epsilon(\alpha+\delta), U(1 / 2, t)=0 .
$$

Thus, we obtained two PDE problems, one for $W$ and another for $U$, which are decoupled and can hence be solved by the standard method of separation of variables. Denoting by $u_{0}(x)$ the initial condition for the original Eq. (42), we can define

$$
Y_{1,0}(x)=u_{0}(x) \text { and } Y_{2,0}(x)=u_{0}(1-x)
$$

for $x \in[0,1 / 2]$. Moreover, we set

$$
W_{0}(x)=Y_{1,0}(x)+Y_{2,0}(x)
$$

and

$$
U_{0}(x)=\left(\frac{1}{2}+\epsilon\right) Y_{1,0}(x)-\left(\frac{1}{2}-\epsilon\right) Y_{2,0}(x) .
$$

Then, by a standard computation, we find

$$
W(x, t)=2(\alpha+\delta)+\sum_{n=0}^{\infty} A_{n} e^{-\alpha_{n}^{2} t / 2} \sin \left(\alpha_{n} x\right)
$$

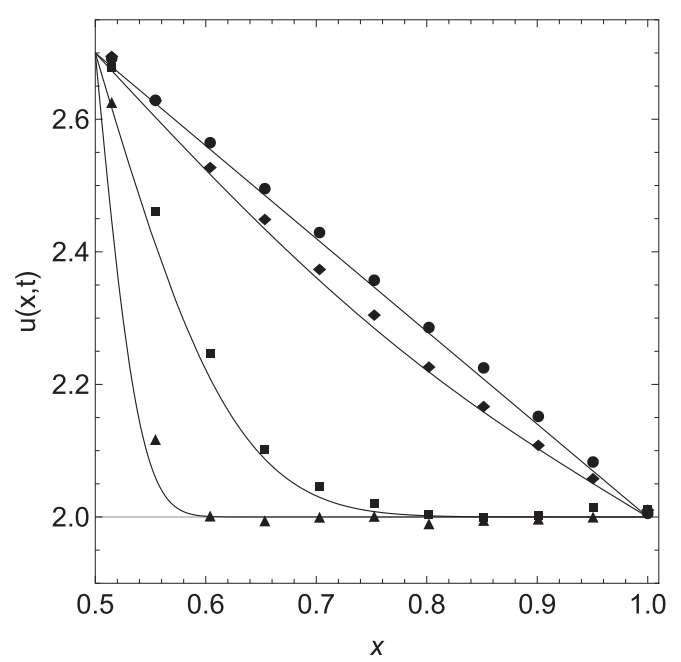

FIG. 7. Comparison between the exact solution of the hydrodynamic problem (49)-(51) and the Monte Carlo measure of the time-dependent density profiles, averaged over a set of different realizations of the stochastic process. The parameters of the simulation are $\alpha=0.5, \delta=1$, $\epsilon=0.4$, and $R=50$. The profiles are plotted at times $t=0.001$ (triangles), $t=0.01$ (squares), $t=0.1$ (diamonds), and $t=0.5$ (circles). The gray and the black solid lines denote, respectively, the initial condition and the exact solution at the corresponding times. The profiles in the regions $[0,1 / 2]$ and $[1 / 2,1]$ are displayed in two separate panels to optimize the resolution of the plots. 

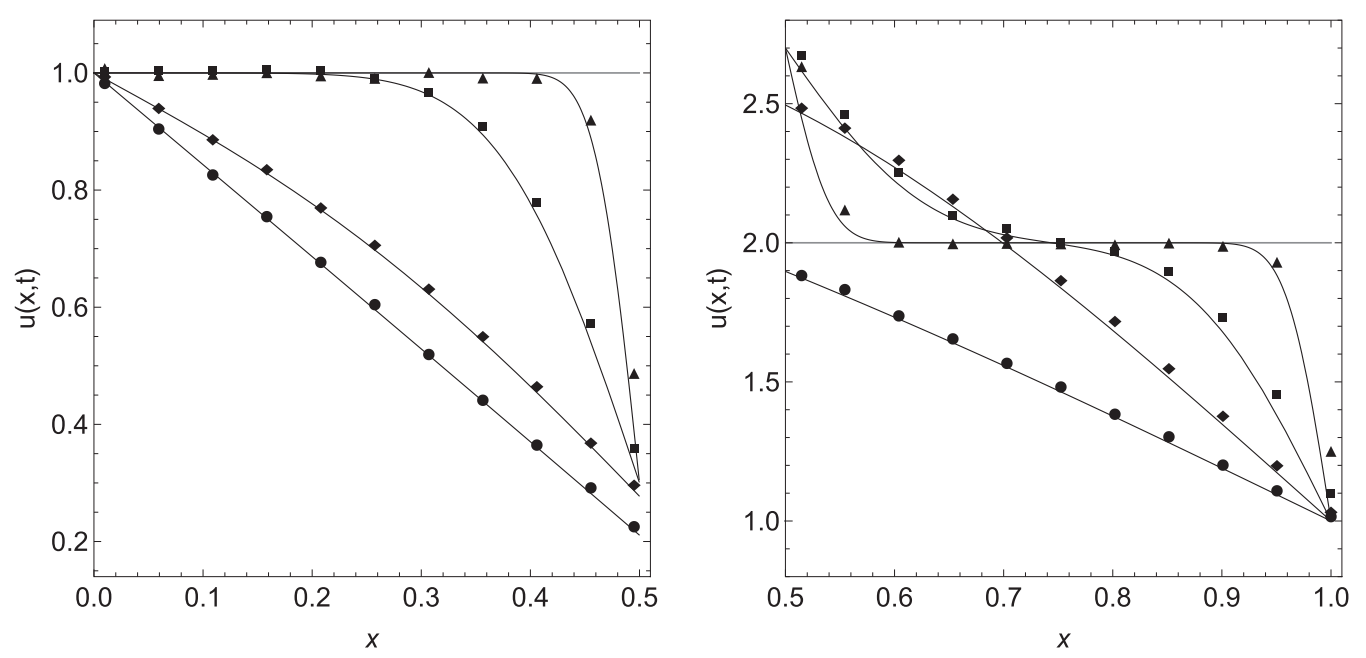

FIG. 8. Comparison between the exact solution of the hydrodynamic problem (49)-(51) and the Monte Carlo measure of the time-dependent density profiles, with $\alpha=\delta=0.5, \epsilon=0.4$, and $R=50$. Symbols are the same as those shown in Fig. 7.

with $\alpha_{n}=(1+2 n) \pi$ and

$$
A_{n}=4 \int_{0}^{1 / 2}\left[W_{0}(x)-2(\alpha+\delta)\right] \sin \left(\alpha_{n} x\right) d x
$$

for $n=0,1, \ldots$ For the function $U$ we find

$$
\begin{aligned}
U(x, t)= & (1-2 x)[\alpha-\delta+2 \epsilon(\alpha+\delta)] \\
& +\sum_{n=0}^{\infty} B_{n} e^{-\beta_{n}^{2} t / 2} \sin \left(\beta_{n} x\right)
\end{aligned}
$$

with $\beta_{n}=2 n \pi$ and

$$
B_{n}=4 \int_{0}^{1 / 2}\left[U_{0}(x)-(1-2 x)[\alpha-\delta+2 \epsilon(\alpha+\delta)] \sin \left(\beta_{n} x\right)\right] d x
$$

for $n=0,1, \ldots$ Solving the equations that define $W$ and $U$ with respect to $Y_{1}$ and $Y_{2}$ we find

$$
Y_{1}(x, t)=\left(\frac{1}{2}-\epsilon\right) W(x, t)+U(x, t)
$$

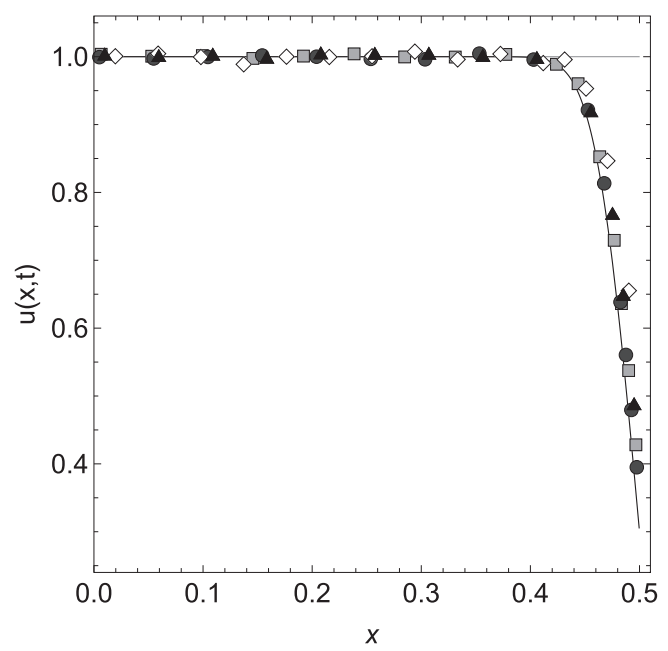

and

$$
Y_{2}(x, t)=\left(\frac{1}{2}+\epsilon\right) W(x, t)-U(x, t) .
$$

Finally, we get the solution of the original problem:

$$
u(x, t)=\left\{\begin{array}{ll}
Y_{1}(x, t) & \text { for } x \in[0,1 / 2] \\
Y_{2}(1-x, t) & \text { for } x \in[1 / 2,1]
\end{array} .\right.
$$

We now test numerically the solution (49)-(51). We consider the hydrodynamic problem in the case $\alpha=0.5$ and $\delta=1$ in Fig. 7 and $\alpha=\delta=0.5$ in Fig. 8. In both figures $\epsilon=0.4$ and the initial datum is $u_{0}(x)=1$ for $x \in[0,1 / 2]$ and $u_{0}(x)=2$ for $x \in[1 / 2,1]$. The density profile is plotted at different macroscopic times and is compared with the numerical estimate.

The numerical solution is constructed as follows: a set of $5 \times 10^{5}$ independent realizations of the stochastic process is constructed by running different Monte Carlo simulations started from the same initial datum (the one also used for the

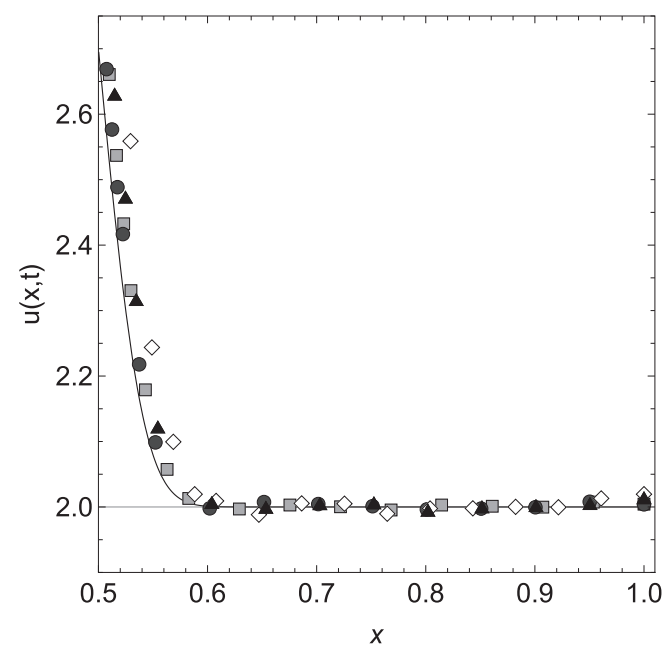

FIG. 9. Comparison between the exact solution of the hydrodynamic problem (49)-(51) (black solid lines) and the Monte Carlo measure, with $\alpha=0.5, \delta=1.0, \epsilon=0.4$, at time $t=0.001$, for different volume sizes: $R=25$ (empty diamonds), $R=50$ (black triangles), $R=75$ (gray squares), and $R=100$ (dark gray circles). The gray solid lines denote the initial condition. 
analytical solution) and by varying the seed of the random number generator routine. Then the profile corresponding to a certain fixed macroscopic time is obtained by averaging over all the different realizations of the process. Finally, the numerical profile is plotted after rescaling the space microscopic variable as $x /(2 R+1) \rightarrow x$, and the very good match illustrated in Figs. 7 and 8 is found.

It should be observed that, in both Figs. 7 and 8, the Monte Carlo results display some (little) discrepancies with respect to the theoretical behavior indicated by the solid lines. These are fluctuations stemming from finite size effects.

Indeed, fixed the initial datum, averaging over a (large enough) set of different realizations of the process corresponds to considering the expectation $\mathbb{E}_{\mu_{R}^{t}}\left[n_{x}(t)\right]$ with respect to a probability measure $\mu_{R}^{t}$ associated with the stochastic process at time $t$. We recall, then, that the hydrodynamic behavior holds in the limit $R \rightarrow \infty$. More precisely, one introduces the empirical density [34]

$$
\pi_{R}^{t}(n)=\frac{1}{2 R+1} \sum_{x \in \Lambda} n_{x}(t) \delta_{x},
$$

where $\delta_{x}$ is the delta measure. From Eq. (52) one finds that, for any continuous function $f: \Omega_{R} \rightarrow \mathbb{R}$, it holds

$$
\int_{\Omega_{R}} f d \pi_{R}^{t}(n)=\frac{1}{2 R+1} \sum_{x \in \Lambda} n_{x}(t) f(x) .
$$

One says, then, that a sequence of probability measures $\mu_{R}^{t}$ on $\Omega_{R}$ is associated with a density profile $u(x, t)$ if for any continuous function $f$ and for any $\epsilon>0$ it holds

$$
\lim _{R \rightarrow+\infty} \mathbb{E}_{\mu_{R}^{t}}\left[\mathbf{1}_{\left|\int_{\Omega_{R}} f d \pi_{R}^{t}(n)-\int_{\Omega_{R}} f(x) u(x, t) d x\right| \geqslant \epsilon}\right]=0,
$$

where 1 denotes the characteristic function.

In Fig. 9 we show that the match between the solution of the hydrodynamic limit equations and the numerical simulation becomes better and better when the size of the lattice used in the simulations increases. The same situation as the one portrayed in Fig. 7 at the macroscopic time 0.001 is considered and simulations are run for $R=25,50,75,100$. Note that the case with $R=50$ (black triangles) is also the case shown in Fig. 7.

\section{CONCLUSIONS}

A variety of systems, e.g., two-species models, particle or spin models undergoing a phase transition, or queuing network models, are known to exhibit uphill currents. In this paper we prove that the phenomenon of uphill diffusion can also be observed in the simplest and, somehow, paradigmatic transport model, namely, the 1D ZRP.

Indeed, such a model is proven to show uphill currents in presence of a bias on a single defect site. For an open ZRP in contact with two particle reservoirs at different densities, for sufficiently large volumes the density at the boundaries of the channel depends only on the injection rates and not on the local bias. If the bias is large enough the current changes sign, so that particles typically move uphill, from the reservoir with lower density to the one with higher density. This result is demonstrated both analytically and numerically, with a striking match between the exact and the Monte Carlo results.

We have also investigated the hydrodynamic limit of the model: a heuristic argument yields the structure of the limit problem and provides the matching conditions mimicking the presence of the defect site in the microscopic lattice model. We managed to write the time-dependent solution as a Fourier series and compared it with the evolution of the original ZRP process.

\section{ACKNOWLEDGMENTS}

We thank A. De Masi and E. Presutti for inspiring this work and for the many enlightening discussions. We also thank D. Andreucci and D. Gabrielli for useful discussions on problems related to the derivation of hydrodynamic limits in presence of local discontinuities.
[1] R. B. Bird, W. E. Stewart, and E. N. Lightfoot, Transport Phenomena, 2nd ed. (John Wiley \& Sons, New York, 2001).

[2] H. C. Öttinger, Beyond Equilibrium Thermodynamics (John Wiley \& Sons, New York, 2005).

[3] J. Erlebacher, M. J. Aziz, A. Karma, N. Dimitrov, and K. Sieradzk, Nature (London) 410, 450 (2001).

[4] J. Alvarez-Ramirez, L. Dagdug, and M. Meraz, Physica A 395, 193 (2014).

[5] A. Lauerer, T. Binder, C. Chmelik, E. Miersemann, J. Haase, D. M. Ruthven, and J. Kärger, Nat. Commun. 6, 7697 (2015).

[6] N. Sato and Z. Yoshida, Phys. Rev. E 93, 062140 (2016).

[7] J. Kärger and D. M. Ruthven, New J. Chem. 40, 4027 (2016).

[8] R. Krishna, Chem. Soc. Rev. 44, 2812 (2015).

[9] L. S. Darken, Trans. AIME 180, 430 (1949).

[10] A. De Masi, E. Presutti, and D. Tsagkarogiannis, Arch. Ration. Mech. Anal. 201, 681 (2011).

[11] M. Colangeli, A. De Masi, and E. Presutti, Phys. Lett. A 380, 1710 (2016).
[12] M. Colangeli, A. De Masi, and E. Presutti, J. Stat. Phys. 167, 1081 (2017).

[13] E. Presutti, Scaling Limits in Statistical Mechanics and Microstructures in Continuum Mechanics (Springer, Berlin, 2009).

[14] M. Colangeli, C. Giardinà, C. Giberti, and C. Vernia, arXiv:1708.00751 (2017).

[15] M. Colangeli, A. De Masi, and E. Presutti, J. Phys. A: Math. Theor. 50, 435002 (2017).

[16] M. Barma, Physica A 372, 22 (2006).

[17] S. A. Janowsky and J. L. Lebowitz, J. Stat. Phys. 77, 35 (1994).

[18] E. N. M. Cirillo, M. Colangeli, and A. Muntean, Phys. Rev. E 94, 042116 (2016).

[19] B. Scoppola, C. Lancia, and R. Mariani, J. Stat. Phys. 161, 843 (2015).

[20] E. N. M. Cirillo, O. Krehel, A. Muntean, and R. van Santen, Phys. Rev. E 94, 042115 (2016).

[21] S. Grosskinsky, P. Chleboun, and G. M. Schütz, Phys. Rev. E 78, 030101(R) (2008). 
[22] C. Godrèche and J. M. Luck, J. Stat. Mech. (2012) P12013.

[23] F. Wilczek, Phys. Rev. Lett. 109, 160401 (2012).

[24] A. Shapere and F. Wilczek, Phys. Rev. Lett. 109, 160402 (2012).

[25] M. R. Evans, Braz. J. Phys. 30, 42 (2000).

[26] T. Bodineau, R. Derrida, and J. L. Lebowitz, J. Stat. Phys. 140, 648 (2010).

[27] F. Spitzer, Adv. Math. 5, 246 (1970).

[28] A. De Masi and E. Presutti, Mathematical Methods for Hydrodynamic Limits (Springer, Berlin, 1991).

[29] M. R. Evans and T. Hanney, J. Phys. A: Math. Gen. 38, R195 (2005).
[30] E. Levine, D. Mukamel, and G. M. Schütz, J. Stat. Phys. 120, 759 (2005).

[31] E. N. M. Cirillo, M. Colangeli, and A. Muntean, Multiscale Model. Simul. 14, 906 (2016).

[32] R. Nelson, Probability, Stochastic Processes, and Queueing Theory: The Mathematics of Computer Performance Modeling (Springer, New York, 1995).

[33] C. Kipnis and C. Landim, Scaling Limits of Interacting Particle Systems (Springer, Heidelberg, 1999).

[34] L. Bertini, A. De Sole, D. Gabrielli, G. Jona-Lasinio, and C. Landim, J. Stat. Phys. 107, 635 (2002). 